\title{
Nanoparticle-conjugated nutraceuticals exert prospectively palliative of amyloid aggregation
}

This article was published in the following Dove Press journal:

International Journal of Nanomedicine

\author{
Kiran Reddy Kanubaddi \\ Shin-Han Yang \\ Li-Wei Wu \\ Chia-Hung Lee \\ Ching-Feng Weng
}

Department of Life Science and Institute of Biotechnology, National Dong Hwa University, Hualien 9740I, Taiwan
Correspondence: Ching-Feng Weng Department of Life Science and Institute of Biotechnology, National Dong Hwa University, No I, Sec. 2, Da Hsueh Rd, Shoufeng, Hualien 9740I, Taiwan

Tel +886 38903637

Fax +886 38630255

Email cfweng@gms.ndhu.edu.tw

\begin{abstract}
Alzheimer's disease (AD), an age-related neurodegenerative disease, the most common causes of dementia is a multifactorial pathology categorized by a complex etiology. Numerous nutraceuticals have been clinically evaluated, but some of the trials failed. However, natural compounds have some limitations due to their poor bioavailability, ineffective capability to cross the blood-brain barrier, or less therapeutic effects on AD. To overcome these disadvantages, nanoparticle-conjugated natural products could promote the bioavailability and enhance the therapeutic efficacy of AD when compared with a naked drug. This application generates and implements new prospect for drug discovery in neurodegenerative diseases. In this article, we confer AD pathology, review natural products in clinical trials, and ascertain the importance of nanomedicine coupled with natural compounds for AD.
\end{abstract}

Keywords: Alzheimer's disease, amyloid beta, bioavailability, natural products, nutraceuticals

\section{Introduction}

Alzheimer's disease (AD), a progression and age-related neurodegeneration disease, is a multifactorial pathology categorized by a complex etiology. $\mathrm{AD}$ is increasingly documented as one of the most vital medical problems affecting the elderly and is the most common type of dementia. The symptoms of AD include progressive memory loss, cognitive impairment such as difficulty solving problems, and disorientation in time and space, among others in an aging population that causes a severe damage of cholinergic neurons in a particular area of the brain, that is, hippocampus. Remarkably, $\mathrm{AD}$ is a prominent cause of death in the USA since 2013; mortality of advanced stage AD increase by $11 \%$ per year. 2016 statistics of Medicaid for the AD predicted to be 19 times more for recipients $>65$ years, who do not have the following symptom. ${ }^{1}$ Apart from the disease itself, it is the burden of care cost that seriously jeopardizes the health and financial security of the patient's family. Of note, three hallmarks of AD pathology are beta-amyloid $(\mathrm{A} \beta)$ accumulations, tau phosphorylation, and inflammation that have been postulated. ${ }^{2}$ The molecular pathology of AD illustrates the abnormal shearing of $\beta$ - and $\gamma$-secretase resulting in $A \beta$ accumulation (Figure 1). 1) The accumulation of $A \beta$ : $A \beta$ accumulation is caused by inaccurate cleavage of the amyloid precursor protein (APP) with $\beta$ - and $\gamma$-secretase; ${ }^{2}$ and subsequently, the massive accumulation of $\mathrm{A} \beta$ mainly formed the plaques. Recently, the hallmarks of molecular features in senile plaque are the unfolding/misfolding of specific proteins/peptides. A $\beta$ peptide consequently becomes susceptible to aggregate into toxic assemblies and deposits that are a crucial histopathological feature, neurofibrillary (tau) tangles, synapse loss, ROS production, and extensive oxidative stress. ${ }^{3}$ This $A \beta$-induced oxidative stress is demonstrated via several clues such as protein oxidation, lipid peroxidation, free 


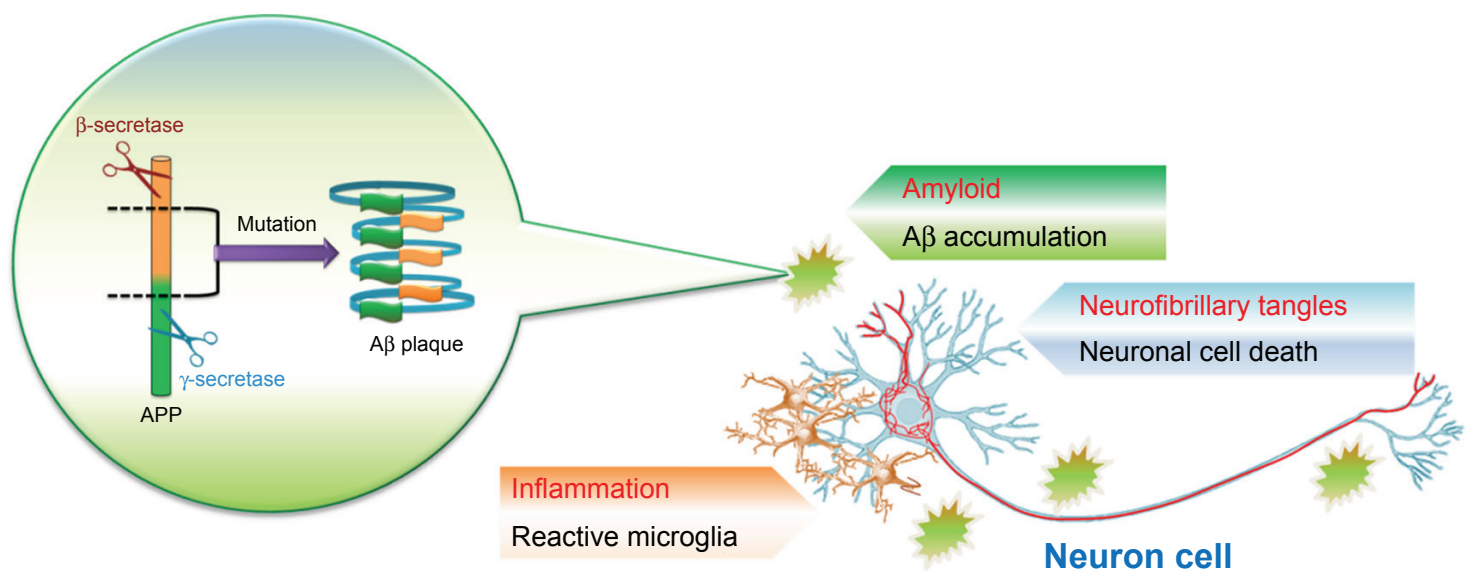

Figure I Alzheimer's disease pathology includes the abnormal shearing of $\beta$ - and $\gamma$-secretase resulting in $A \beta$ accumulation. Notes: Phosphorylation of tau protein causes nerve entanglement. Inflammation and phagocytosis are induced by microglia. Abbreviations: A $\beta$, beta amyloid; APP, amyloid precursor protein.

radical formation, DNA oxidation, and neuronal cell death. ${ }^{4}$ Novel metabolic processing events such as $\delta$-secretase and $\beta$-secretase have revealed that they generate previously uncharacterized APP metabolic fragments with the impending to be involved in AD pathogenesis. ${ }^{5}$ The accumulation of amyloid plaque in the brain due to the accumulation caused by $A \beta 1-40$ and $A \beta 1-42$ peptides, $A \beta \quad 1-40$ and $A \beta$ 1-42 inhibit endocytosis, and $A \beta$ 1-40 can inhibit lysosomes. The uptake led to increased accumulations of $A \beta 1-42, A \beta 1-40$, and $A \beta$ 1-42, which also perturbs neuronal trafficking and vesicular dynamics. ${ }^{6}$ 2) Hyperphosphorylation of tau protein: tau protein is a microtubule-associated protein expressed in the axons and soma of nerve cells. ${ }^{7}$ Phosphorylation of tau protein is due to phosphorylation of serine and threonine sites for the tau protein. ${ }^{8}$ The tau protein hyperphosphorylation led to neurofibrillary tangles because cognitive impairment may be a more direct effect than $A \beta$ accumulations. ${ }^{9}$ And 3) microglia communication driving for AD-related functional impairments. In $\mathrm{AD}$, microglia aging is owing to cytokines that complement extracellular vesicles. The consequence of these changes includes augmented inflammation, reduced phagocytosis, and declined motility. ${ }^{10}$ Furthermore, aged microglia would augment the levels of IL-1 $\beta$, tumor necrosis factor- $\alpha$, and IL-6. ${ }^{11}$ Microglia enhances inflammatory responses and inhibits phagocytosis that presumably might be increased by age and led to decreased synaptic plasticity. ${ }^{12}$

Clinically, the early stages of AD have been treated by using acetylcholinesterase inhibitors. ${ }^{13}$ As we know, there are five Food and Drug Administration-approved treatment medicines for the management of AD, which all offer symptomatic benefits. Tacrine, donepezil, galantamine, and rivastigmine are acetylcholinesterase inhibitors and are
N-methyl-D-aspartate receptor antagonists. ${ }^{14}$ Unfortunately, none of these drugs could cure or delay the commencement of $\mathrm{AD}$ due to various causes of dementia and neuropathology in many patients. ${ }^{15}$ This failure could be owing to the unclear underlying pathways of AD. For example, the accumulation of neurotoxic $A \beta$ peptides in the brain exemplifies a pathogenic hallmark of $\mathrm{AD}$, which is the most general form of dementia in an aging population. ${ }^{16}$ It was found that the decreased clearance rather than the production of $A \beta$ is the primary formation of the deleterious $A \beta$ plaques in the brain. ${ }^{17}$ The lessened removal of $\mathrm{A} \beta$ from the brain into the blood can be moderately attributed to the dysfunction of P-gp function, leading to the progression of AD. ${ }^{18-20}$ Furthermore, it has been shown that $\mathrm{A} \beta$ can downregulate the P-gp expression along with other transporters, which consequently results in further accelerated neurodegeneration. ${ }^{21}$ Hence, it has been suggested that the increased $A \beta$ clearance from the brain by restoring blood-brain barrier (BBB) P-gp function to diminish A $\beta$ brain accumulation is a new strategy for medical treatment in the early stage of AD. ${ }^{22,23}$ Unexpectedly, as of 2017, Alzheimer's treatment drugs, verubecestat and solanezumab, have been discontinued in phase III clinical trials of A $\beta$ protein, but they have also led to the worldwide pathogenesis of $\mathrm{AD}$, and new candidates require more attention to accelerate drug discovery for clinical use. ${ }^{24-26}$ Therefore, the devotion of discovering agents against amyloidosis has turned to the search from natural compounds for meeting this demand.

\section{Natural compounds act as nutraceuticals}

The alternative approach instead of standard medical treatments in clinic for treating the patients is broadly recognized by using chemical derivatives, ${ }^{27-30}$ natural sources including 
herb, and traditional medicine even natural compounds. In this article, we mainly focus on the benefits of natural compounds as an alternative use for neurodegenerative disease to confer this theme. Natural compounds are found abundantly in nature, particularly in daily foods, such as edible vegetable and fruit juices, green tea, wine, turmeric, and even cigarettes. ${ }^{31}$ All of which contain antioxidative substances, especially polyphenols that act as both ROS scavengers and transition metal chelators. ${ }^{32}$ Their antioxidant effects are naturally linked to anti-AD potential. In addition, their evolutional structure has made them become beneficial enzyme activators, channel openers, and receptor agonists. ${ }^{33}$ Owing to the antioxidant activity in higher plants, consideration has improved about the defending activity of its natural antioxidants against chronic disorders caused by the oxidative process. ${ }^{34}$ Antioxidants and nutrition have long been deliberated as an approach to alleviate $\mathrm{AD}$ progression. Numerous investigations have exposed that folic acid, vitamin B12, choline, vitamin C, vitamin K3, vitamin D3, vitamin E, zinc, selenium, s-ethyl cysteine, s-propyl cysteine, citicoline, rivastigmine, memantine, tea polyphenol, curcumin, caffeine, $\alpha$-lipoic acid, $\mathrm{N}$-acetylcysteine, and dietary polyphenols are able to interact with gene expressions and epigenetic mechanisms. ${ }^{35-39}$ A growing evidence also suggests that epigenetic alterations are elicited by dietary nutrients that possess an imperative role in health and the preventative occurrence of some diseases, particularly neurodegenerative disorders. Assured natural dietary polyphenolic phytochemicals have paid extensively recent attention as alternative candidates for AD therapy. In particular, curcumin, resveratrol, piperine and spices, extra virgin olive oil, red wine, red berries, and green tea catechins contained antiamyloidogenic, antioxidative, and anti-inflammatory properties that have been postulated to have the preventative potential for $\mathrm{AD} .40,41$ Therefore, the attention of discovering agents against amyloidosis has turned to the search of natural compounds. Prominently, natural compounds have often been demonstrated to have better pharmacological properties than synthetic small molecules, especially with regard to less toxicity and good absorption. ${ }^{33}$ Emergent studies revealed that some natural compounds isolated from Chinese herbs could be administered for disease or cancer therapy ${ }^{42,43}$ Based on these successes, we have listed the natural substances of the most studied on neuroprotective and reviewed the following natural compounds: 1) scyllo-inositol, 2) curcumin, 3) Ginkgo biloba extract, 4) resveratrol, and 5) epigallocatechin gallate along with their current clinical trials on $\mathrm{AD}$ treatment (Table 1). And the chemical structures of four discussed compounds are also exhibited underneath.

\section{Scyllo-inositol}

Scyllo-inositol, an inositol stereoisomer, is abundant in the coconut palm. Numerous studies have shown that scyllo-inositol can bind and inhibit $A \beta$ aggregation and the formation of $A \beta$ fibrils in vitro. ${ }^{44}$ In the TgCRND8 mice model, the amyloid pathology has delayed in a dose-dependent fashion. ${ }^{45}$ The phenomenon of neuronal autophagy in TgCRND8 model has also been explored. ${ }^{46}$ The clinical trial assessing the safety and efficacy of multiple oral dosages of scylloinositol has applied to the treatment of AD, which started in 2007. However, serious adverse events occurred, including the death of nine patients, and have forced the company to stop the highest two doses and retain $250 \mathrm{mg}$ scyllo-inositol twice a day until 2017. The differences between the $250 \mathrm{mg}$

Table I Summary of natural products in clinical trial

\begin{tabular}{|c|c|c|c|c|}
\hline Compounds & Source & Pathway & Trial status & $\begin{array}{l}\text { Reference/ } \\
\text { ClinicalTrials.gov ID }\end{array}$ \\
\hline Acetyl-L-carnitine & Meat & Antioxidative stress & Phase II (failed) & 136,137 \\
\hline Bryostatin I & Bugula neritina & Reduce $A \beta$ formation & Phase II (ongoing) & NCT0243।468 \\
\hline Curcumin & Curcuma longa & Reduce $A \beta$ formation & Phase II (failed) & 78/CT000997I0 \\
\hline Docosahexaenoic acid & Fish oil & Reduce $A \beta$ formation & Phase II (failed) & 79/NCT00440050 \\
\hline n-Butylphthalide & Ligusticum & Reduce $A \beta$ formation & Phase II (ongoing) & | 38/NCT027| | 683 \\
\hline Pinitol & Sutherlandia frutescens & Inhibit $\gamma$-secretase & Phase II (completed, result not published) & I39/NCT0I928420 \\
\hline Scyllo-inositol & Coconut palm & Reduce $A \beta$ formation & Phase II (evidence insufficient) & $\begin{array}{l}\text { 80/NCT00568776, } \\
\text { NCT00934050 }\end{array}$ \\
\hline Epigallocatechin gallate & Green tea & Antioxidative stress & Phase II (completed, result not published) & I40/NCT0095 I834 \\
\hline Ginkgo biloba extract & G. biloba & Antioxidative stress & Phase III (failed) & $81,82 / \mathrm{NCT} 00010803$ \\
\hline Resveratrol & Grape & Reduce $A \beta$ formation & Phase II (evidence insufficient) & I4I/NCT0I504854 \\
\hline Huperzine A & Huperzia serrata & Reduce $A \beta$ formation & Phase II (unknown) & 142 \\
\hline
\end{tabular}


scyllo-inositol and placebo groups were not significant for the coprimary or secondary endpoints.

\section{Curcumin}

Curcumin (diferuloylmethane), one component of turmeric, is isolated from the rhizome of Curcuma longa and abounds in ginger family (Zingiberaceae). Numerous studies have focused on the various facets of curcumin due to its antioxidant and anti-inflammatory properties; curcumin also plays a significant advantageous and pleiotropic regulatory role in various pathological conditions which including hyperglycemia, oxidative stress, and cancer, ${ }^{47}$ cardiovascular disease, AD, anti-inflammation, ${ }^{48}$ neurological disorders, ${ }^{49}$ and various malignant diseases. ${ }^{50}$ Apart from these well-known suppressing activities, this natural polyphenolic compound also exerts its profitable effects by mediating different signaling molecules such as transcription factors, chemokines, cytokines, tumor suppressor genes, adhesion molecules, and microRNAs. ${ }^{51}$ Notably, oxidative stress (free radicals) and inflammation are responsible for many human health problems including aging, arthritis, cancer, cardiovascular disease, diabetes, neurological disorders, AD, Parkinson's disease, mild cognitive impairment, alcohol-induced liver disease, ulcerative colitis, and atherosclerosis. ${ }^{52}$ The neuroprotective effects of curcumin and curcuma oil exert its significant action in the decline of NO-induced peroxynitrite formations and cell apoptosis in the transient middle cerebral artery occlusion (MCAo) model and focal embolic stroke model rat. ${ }^{53,54}$ Furthermore, curcuminoids have been used to perform as latent therapeutic implications for various neurodegenerative diseases. ${ }^{55}$ The mechanism of curcumin for protecting the rat hippocampus combating with the neurotoxicity of homocysteine oxidative stress could be possible by increasing the endogenous defenses against oxidative stress via inhibiting the ROS generation of brain. ${ }^{56}$ In cellular studies, curcumin inhibits cyclooxygenase-2, tumor necrosis factor- $\alpha$, and IL-1 expression. ${ }^{57}$ It downregulates IL-6 signaling via suppressing phosphorylation of STAT3 ${ }^{58}$ and inhibits $A \beta$-induced microglial inflammation through modulating ERK1/2 and p38 signaling pathways. ${ }^{59}$ Curcumin can serve as a neuroprotective reagent through various signal pathways: acting against endothelin-1-mediated cell death (decreasing proapoptotic signaling) via blocking an increase in c-Jun levels in primary hippocampal neurons ${ }^{60}$ and inhibiting $A \beta$ generation through induction of autophagy by downregulating PI3K/Akt/mTOR signaling pathway. ${ }^{61}$ Furthermore, chronic application of curcumin might ameliorate AD-related cognitive deficits and upregulate brain-derived neurotrophic factor-ERK signaling in the hippocampus. ${ }^{62}$
There was a marked reduction in the production of $\mathrm{A} \beta$ when human neuroblastoma cells were treated with curcumin. ${ }^{63}$ The aggregation of $A \beta$ in a rat model of $A D$ was also reduced after oral curcumin administration, as well as with the improvement of cognitive impairment in a spatial learning and memory test, suggesting that curcumin could be a candidate for treating AD. Currently, there are three clinical trials investigating the effect of anti-A $\beta$ formation, but all of them failed to exhibit clinical or biochemical evidence of efficacy for AD. Even the underlying mechanisms of curcumin will be hampering the formation and promoting the disaggregation of $\mathrm{A} \beta$ plaques, mitigating the hyperphosphorylation of tau and augmenting its clearance, binding copper, lowering cholesterol, amending microglial activity, inhibiting acetylcholinesterase, regulating the insulin signaling pathway, and is an antioxidant as well. ${ }^{64}$

\section{EGb-76I}

EGb-761 is made of ginkgo leaves, one dry extract that is adjusted to contain ginkgo flavonoids and terpene lactones. ${ }^{65}$ In the cellular study, EGb-761 decreases free cholesterol levels and neuronal $A \beta$ production, and the level of Alzheimer's amyloid precursor in the brain is also decreased. ${ }^{66}$ It has been found in rescuing impaired mitochondrial function and improving neuronal energy supply. ${ }^{67}$ The protective and rescuing abilities of EGb-761 are attributable to the antioxidant properties and the ability to inhibit NO-stimulated protein kinase $\mathrm{C}$ activity. ${ }^{68}$ The increased production of toxic mediators such as hydrogen peroxide and platelet-activating factor in the brain may be critical in the pathological mechanism of neurodegenerative diseases particularly AD and is blocked by EGb-761. ${ }^{69}$ In the current study, the dopamine level increased in the rat prefrontal cortex after EGb-761 administration, which implied a benefit for memory function. ${ }^{70}$ Although the active ingredients of EGb-761 have not been explored, it still has the potential to treat or prevent AD. Nevertheless, the clinical trial for evaluating G. biloba's ability to abrogate memory loss or delay dementia still leads to negative outcomes.

\section{Resveratrol}

Resveratrol (Res), a phytochemical, has been found in many plant species such as herbs, berries, grapes, and peanuts. Res exhibits diverse biochemical properties, such as antiplatelet $^{71}$ and anti-inflammatory properties. ${ }^{72}$ It has been shown to reduce oxidative stress and stabilize mitochondria through regulating Sirt1 pathway. Res is reported as a potential nutraceutical agent for $\mathrm{AD}$; evidence indicates that Res alleviated Tau hyperphosphorylation at Ser396 site and oxidative damage in rat hippocampal slices exposed 
to vanadate via ERK1/2 and glycogen synthase kinase-3 $\beta$ signaling cascades. ${ }^{73}$

Res retreating $\mathrm{A} \beta$-induced learning and memory disorder may involve the regulation of neuronal inflammation and apoptosis via phosphodiesterase-4-related Cyclic adenosine monophosphate (cAMP)-CREB-brain-derived neurotrophic factor signaling ${ }^{74}$ and inhibit $A \beta$-induced neuronal apoptosis through reversion of silent information regulator 1 activity and subsequently the downregulation of Rho-associated kinase 1 signaling pathway. ${ }^{75}$ Recently, one study showed that resveratrol changed three $\mathrm{A} \beta$ conformers into nontoxic alternations, suggesting that Res could mediate $A \beta$ toxicity. ${ }^{76}$ It also lowered $A \beta$ levels in $\operatorname{Tg} 2576$ mice by stimulating nonamyloidogenic processing of APP. ${ }^{77}$ According to its amyloidogenic-delaying and antioxidant effects, Res could be helpful in fighting AD. In recent years, four clinical trials assessing the effects on AD were established. However, none of them succeeded in different phases due to insufficient evidence and nonsignificant outcomes. Among these natural compounds with high neuroprotective effects that have broadly studied, have extensively used as an antioxidant for free radical scavenger. Nevertheless, the antioxidant and anti-A $\beta$ formation activity of these natural compounds in vitro/in vivo failed to be well translated into therapeutic effects for patients with $\mathrm{AD}$ in clinical trial. ${ }^{78-82}$ In fact, the therapeutic success of many pharmaceutical remain moderate because of their low penetration across BBB, which limits their targeting. Therefore, carrying sufficient drugs through BBB becomes a long-term issue. Despite such phenomenal advances in medicinal applications, the clinical implication of native curcumin is hindered due to poor aqueous solubility, physicochemical instability, low compatibility, rapid metabolism, and poor pharmacokinetics. ${ }^{83}$ Therefore, low compatibility and low bioavailability may hinder its usefulness as a therapeutic agent. Once the defy of low bioavailability is overcome, curcumin-based medications for AD might be in the prospect.

In view of nanoparticles posture that is extensively researched in many fields, that provides this new approach for facilitating the efficiency of disease therapy particular in neurodegenerative diseases including AD. According to the abovementioned five natural compounds for $\mathrm{AD}$ treatment in clinical trial, however, no extensive studies on the potential pharmaceutical applications of combinations of Epigallocatechin gallate, EGb-761, and Scyllo-inositol with nanoparticles and their synergistic effects have been performed. Furthermore, conventional strategies failed to treat $\mathrm{AD}$ in clinical trials, partly due to the poor solubility, low bioavailability, and ineffectiveness of the tested natural compounds to cross $\mathrm{BBB}$. We will plausibly deliberate the beneficial effect or potency of nanocarriers conjugated with two natural compounds (resveratrol and curcumin) and feasibly postulate a potential natural candidate quercetin to complement a new expectation for AD treatment.

\section{Nanoparticles conjugated natural products for $A D$}

The nanocarrier formulations, which are featured in its particle size, adjustable component, and surface charges, have demonstrated to encapsulate commercial drugs or molecules. ${ }^{84,85}$ The technique may provide an alternative way to augment drug transport through the BBB in neurodegenerative disorders.$^{86}$ In addition, by conjugating specific antibodies, nanoparticle may target specific regions. ${ }^{87}$ Therefore, to address natural compounds for the brain via nanocarriers becomes a popular topic. Next, we describe nanoparticle as a successful delivery carrier in the development of $\mathrm{AD}$ therapeutics and diagnostics.

\section{Nanoparticle-conjugated curcumin Gold}

Gold nanoparticles (AuNPs) are one of the major components of bionanotechnology applications. ${ }^{88}$ Special properties of AuNPs are low toxicity, highly biocompatible, ${ }^{89}$ well functionalization, and plasmon-based strong optical characters that are used for detection/imaging. ${ }^{90-92}$ In addition, curcumin is hydrophobic in nature that shows less solubility limited to therapeutic applications; ${ }^{93}$ however, some results show that curcumin is conjugated with hyaluronic acid and polymers on the surface of AuNPs to improve bioavailability. ${ }^{94,95}$ In one previous study, curcumin was modified by monocarboxylic acid conjugated with primary amine-terminated silica-coated AuNPs that was applied to evaluate water solubility of conjugated complex (Figure 2A). In nanoparticle conjugated curcumin becomes more water-soluble and can efficiently interact with amyloid protein/peptide, offering enhanced performance in inhibiting amyloid fibrillation and dissolving amyloid fibrils. Curcumin monocarboxylic acid derivative was measured using hen egg white lysozymes compared with naked curcumin and Au-curcumin modification in which Aucurcumin derivatives show high inhibits of $A \beta$ fibrillation and dissolve/disintegrate $A \beta$ fibrils. ${ }^{96}$ After this conjugation, the data confirmed to improve the water solubility of curcumin for a promising therapeutic approach. However, unmodified curcumin has poor sensitivity, specifically it is difficult to penetrate the blood-brain barrier, researchers choosing 
A

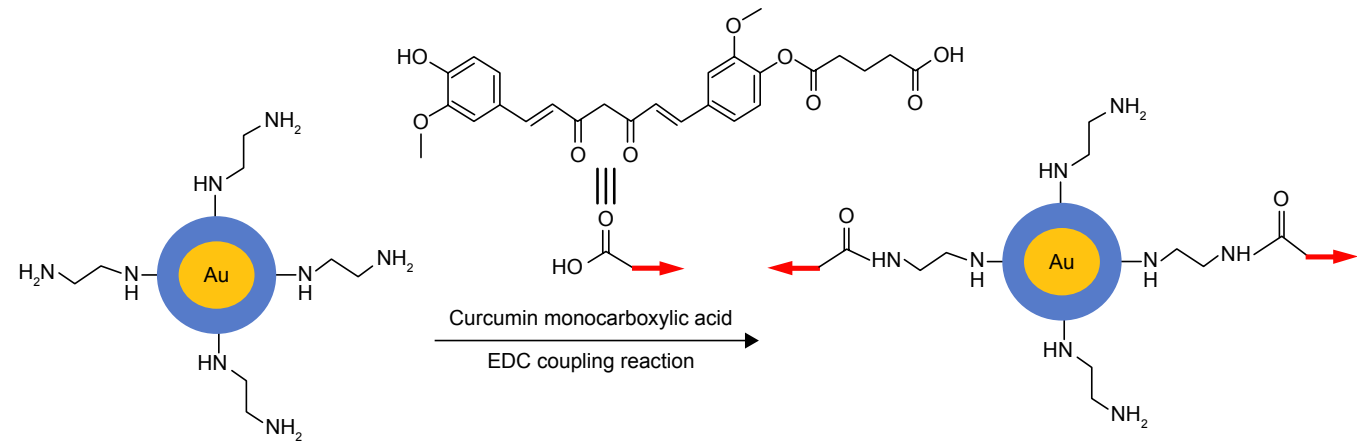

B Brain-targeted lipid nanoparticle

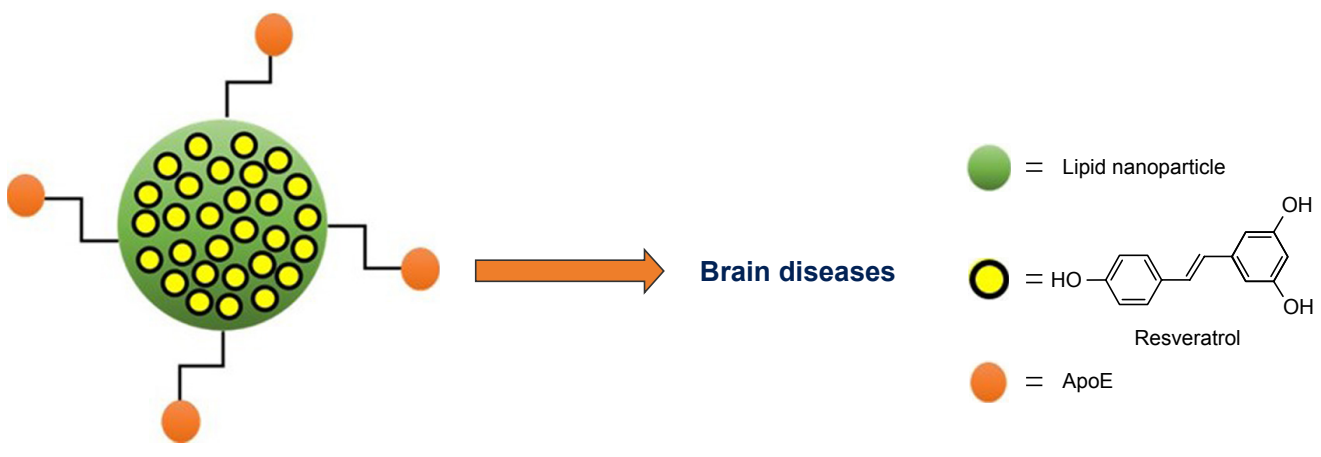

C
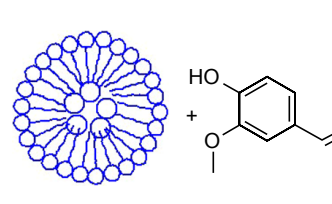<smiles>C=CC(=O)CC=CC(=O)C=CCOC</smiles>

Curcumin
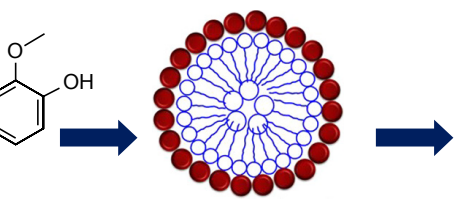

Diagnosis and treatment

Nanoliposome

Nanoliposome-conjugated curcumin

D

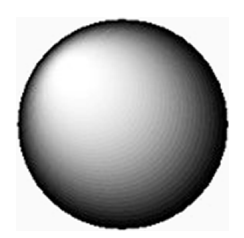

Graphene oxide

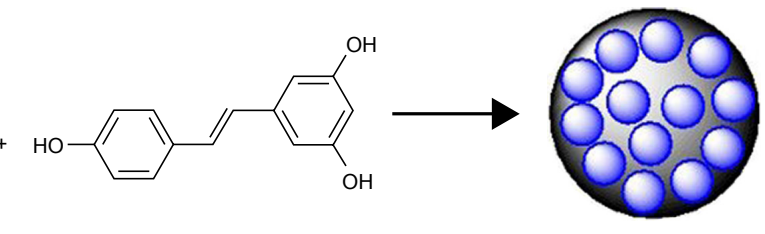

Resveratrol

\section{Res@GO}

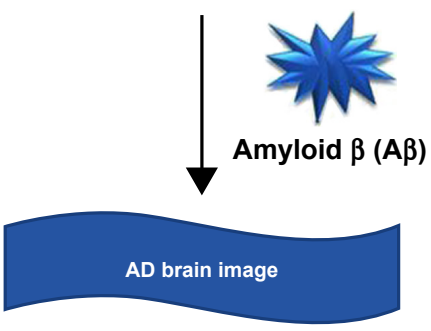

Figure 2 The applications of natural compounds conjugated with nanoparticles.

Notes: (A) Synthesis of curcumin-functionalized gold nanoparticles by using EDC coupling. @ 20I4WILEY-VCHVerlag GmbH \& Co. KGaA,Weinheim. reproduced from Palmal S, Maity AR, Singh BK, Basu S, Jana NR. Inhibition of amyloid fibril growth and dissolution of amyloid fibrils by curcumin-gold nanoparticles. Chemistry. 20I4;20(20):6I84-6I9I.96 (B) Resveratrol-conjugated solid lipid nanoparticles. Copyright @2016 Neves et al. Reproduced from Neves AR, Queiroz JF, Reis S. Brain-targeted delivery of resveratrol using solid lipid nanoparticles functionalized with apolipoprotein E. J Nanobiotechnology. 2016;14(I):27.131 (C) Nanoliposomes conjugated with a curcumin derivative. (D) Simple construction of Res@GO for the rapid fluorogenic probing of A $\beta$. Adapted with permission from He XP, Deng Q, Cai L, et al. Fluorogenic resveratrol-confined graphene oxide for economic and rapid detection of Alzheimer's disease. ACS Appl Mater Interfaces. 20I4;6(8):5379-5382. Copyright 20I4 American Chemical Society. ${ }^{132}$

Abbreviations: A , beta amyloid; AD, Alzheimer's disease; APP, amyloid precursor protein; APoE, apolipoprotein E; EDC, N-(3-dimethyl amino propyl)-N-ethyl carbodiimide; GO, graphene oxide. 
alternatively, the polyhydroxyl substituted squaraine dyes 1-3 under investigation act as effective protein-labeling and destabilizing agents of the protein amyloidogenesis as well. ${ }^{97}$ Undeniably, AuNPs were more expensive than silver (Ag). Recently, fluorescence quenching by lipid encased Ag nanoparticle is performed to discover that membrane-inserted $\mathrm{A} \beta$ oligomers have a preferred molecular orientation..$^{98}$ These new approaches might be useful for accelerating the investigation of molecular mechanism in $\mathrm{AD}$ treatment to discover new drug for therapy.

\section{Nanogels}

Hydrogels are hydrophilic three-dimensional polymers ${ }^{99}$ that are manipulated into nano dimensions called nanogels. ${ }^{100}$ The diameter of nanogels varies from 10 to $300 \mathrm{~nm}$ and depends on component ratio. ${ }^{101,102}$ This nanogel has special features such as high loading capacity and stability and variable environment factors (ionic strength, $\mathrm{pH}$, temperature) that provide a novel platform for drug delivery. Additionally, it has a microheterogeneous structure, small size, and high surface to volume ratio. ${ }^{103,104}$ Furthermore, nanogels create hydrophobic interactions that can increase oral and brain delivery of lowmolecular-weight drugs and biomacromolecules. ${ }^{104}$ Nanogelconjugated poor water-soluble drugs such as curcumin could improve their bioavailability. ${ }^{105,106}$ Another drawback of curcumin was unstable and it is easy to be degraded in alkaline aqueous solutions. ${ }^{107,108}$ To overcome this limitation, multifunctional nanogels conjugated with curcumin giving the improvement of stability and the protection of curcumin degradation as well, making curcumin more potentially bioavailable for therapeutic applications in AD that could more efficiently inhibit $\mathrm{A} \beta$ fibrillogenesis and amyloid cytotoxicity than those of nonconjugation curcumin. ${ }^{106}$

\section{Polymers}

Polymer nanoparticles are characterized as solid and colloidal whose particles size ranges from 10 to $1,000 \mathrm{~nm} \cdot{ }^{109,110}$ They show excellent properties such as biocompatibility and biodegradability for therapeutic applications. ${ }^{11,112}$ Furthermore, hydrophobic drugs such as curcumin conjugated or encapsulated with polymers could enhance the curcumin bioavailability. ${ }^{113-115}$ In addition, curcumin encapsulation with poly(butyl)cyanoacrylate nanoparticles was conjugated on outer surface with apolipoprotein E3 to promote therapeutic efficacy against A $\beta$-induced cytotoxicity in SH-SY5Y neuroblastoma cells; interestingly, poly(butyl)cyanoacrylate-loaded curcumin shows high therapeutic efficacy compared with nonencapsulation curcumin. ${ }^{116}$ Water-soluble poly(lactide-co-glycolide) (PLGA) nanoparticle loaded curcumin covalently conjugated with the Tet-1 peptide is able to destroy amyloid aggregation and enhance antioxidative and noncytotoxic properties of this complex are examined by in vitro studies. ${ }^{117}$ Another interesting fact is that PLGA nanoparticles encapsulated with curcumin in the ratio of 50:50 (w/w) are able to suppress or cease phosphorylation of protein kinase $\mathrm{B}$ (Akt) and tau proteins in human neuroblastoma SK-N-SH cells. The formulations have immense applications in pharmacology and have also been applied to treat neurodegenerative diseases such as $\mathrm{AD} .{ }^{118}$ The encapsulation of curcumin with PLGA nanoparticles in a diameter that varies from 80 to $120 \mathrm{~nm}$ is nontoxic to the SK-N-SH cells and protects neurons from oxidative damage in $\mathrm{AD} .{ }^{119}$ In recent studies, the functionalization of PLGA nanoparticles with glutathione (GSH) was loaded with curcumin using click reaction innovative strategy for neuronal cell delivery. ${ }^{120}$

\section{Nanoliposomes}

Liposome is originally derived from two Greek words: "Lipos" meaning fat and "Soma" meaning body. Liposomes were first described in 1960s by Bangham et al ${ }^{121}$ and implicated as a potential drug delivery system in early 1970 s. ${ }^{122,123}$ Liposomes are made up of phospholipids and are selfenclosed to form spheres of lipid bilayers and with an aqueous core in its bilayers. Owing to the side of the hydrophobic and hydrophilic character (in addition to biocompatibility), liposomes are accomplished systems for drug delivery. ${ }^{124}$

Curcumin shows high affinity for $A \beta$ peptide with the fluorescence character, however, extremely low aqueous solubility limits its clinical use. Thereby, curcumin-conjugated nanoliposomes were designed for monitoring the amyloid peptide deposits as they were more stable and appeared as monodisperse. They had nontoxic in vitro properties, downregulated the secretion of $A \beta$ peptide, and partially prevented $\mathrm{A} \beta$-induced toxicity. Additionally, this conjugation firmly marked $\mathrm{A} \beta$ deposits in postmortem brain tissues of $\mathrm{AD}$ patients and APP/PS1 mice. Moreover, curcumin-conjugated nanoliposomes were injected into the hippocampus and the neocortex of APP/PS1 mice; the data showed that curcumin nanoliposomes were specifically stained in the $A \beta$ deposits in vivo. Therefore, curcumin-conjugated nanoliposomes could discover the application of diagnosis and targeted drug delivery in $\mathrm{AD}$ patients (Figure 2C). ${ }^{125}$

Nanoliposomes conjugated with a curcumin derivative formed a planar structure for interaction with $A \beta$ that could be detected by using surface plasmon resonance experiments. The second type of liposomes was conjugated with phospholipid in which the planar structure of curcumin does not show the planner structure. Both types of curcumin-decorated vesicles with diameters between (131-207 nm) and marginally 
negative $\zeta$-potential values based on their lipid composition. Also, they were highly stable lasting up to 20 days. They likewise showed high integrity during incubation in the presence of plasma protein delivery in $\mathrm{AD}$. In surface plasmon resonance experiments, the measurements of the binding of flowing liposomes to immobilized $A \beta 1-42$ revealed that the liposomes exposing to the curcumin derivative (maintaining the planarity) have an extremely high affinity for $A \beta 1-42$ fibrils (1-5 nM), owing to the occurrences of multivalent interactions; whereas nonplanar curcumin did not bind to A $\beta$ 1-42. However, curcumin derivative demonstrated that planar with a high affinity for $A \beta 1-42$ fibrils is taken into considerations as vectors in the targeted delivery of new diagnostic and therapeutic molecules for AD. ${ }^{126}$

\section{Nanoparticle-conjugated resveratrol}

Resveratrol (Res), a polyphenol compound, has shown great significance in therapeutic effects, including anticancer, antioxidation, and anti-inflammation. However, there are some limitations in pharmacokinetic characters such as low aqueous solubility and poor bioavailability. Recently, nanoformulations are viewed as a novel technique for enhancing the pharmacokinetic features as well as enhancing target ability and bioavailability of Res. ${ }^{127}$ Also, Res is able to inhibit the formation and aggregation of $A \beta$ peptides, which are liable for neuronal dysfunction and death associated with $\mathrm{AD}$ due to its ROS-generating action. ${ }^{128}$ When rats received a single intracerebroventricular injection of $A \beta$ 1-42 ( $2 \mathrm{nmol}$ ), and 1 day after $A \beta$ infusion, they were intraperitoneally administered either free Res or (Res)-loaded lipid-core nanocapsules ( $5 \mathrm{mg} / \mathrm{kg}$, each 12 hours) for 14 days. A $\beta$ 1-42infused animals were proved to be a significant impairment of learning memory capacity, which was associated with a significant decrease in hippocampal synaptophysin levels. Furthermore, astrocytes and microglial cells of animals are activated as well as the disturbance in c-Jun N-terminal kinase

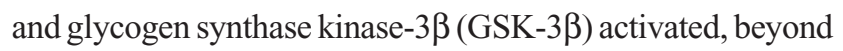
destabilization of $\beta$-catenin levels. These results remarkably reveal that by utilizing lipid-core nanocapsules, Res could protect the deleterious effects of $A \beta$ 1-42 while treatments with Res alone present only partial beneficial effects. The increase of Res concentrations might explain these findings in the brain tissues achieved by lipid-core nanocapsules. ${ }^{129}$ It is noteworthy that $\mathrm{AD}$ treated with grape skin and grape seed extracts increases the inhibitory effect on $A \beta$ aggregation. However, after intravenous injection, Res is rapidly metabolized into both glucuronic acid and sulfate conjugated with the phenolic groups in the liver and intestinal epithelial cells (within $<2$ hours), which are then eliminated. A recent report has demonstrated that solid lipid nanoparticles (SLNs) functionalized with the antitransferrin receptor monoclonal antibody (OX26 mAb) can work as a possible carrier for transporting this extract to target the brain. In human brainlike endothelial cells, experiments illustrate that the cellular uptake of the OX26 SLNs is considerably more efficient than that of normal SLNs and SLNs functionalized with an nonspecific antibody. Consequently, the transcytosis ability of these different SLNs is higher when functionalized with OX26. ${ }^{130}$ On the contrary, Res encapsulation SLNs were functionalized with apolipoprotein $\mathrm{E}$, which can be perceived by the overexpressed $\mathrm{BBB}$ of low-density lipoprotein receptors (Figure 2B). In vitro cytotoxicity of hCMEC/D3 cell line assessed by MTT and LDH assays, the results revealed no toxicity up to $50 \mu \mathrm{M}$ over 4 hours of incubation. The permeability through hCMEC/D3 monolayers showed a significant increase (1.8-fold higher) in Res encapsulation of SLNs functionalized with apolipoprotein E compared with nonfunctionalized ones. ${ }^{131}$

Additionally, Res is performed to detect $\mathrm{AD}$ for increasing an advantageous potential of the real-time probing concerning $\mathrm{A} \beta$ that is closely implicated in $\mathrm{AD}$ or ought to assist higher understanding or monitoring the disease. Res combined with graphene oxide (GO) for the rapid, fluorogenic recognition of A $\beta$. This Res@GO composite could capture both A $\beta$ monomers and fibers in a physiological buffer solution within 3 minutes that have been proved in this study. And Res@GO composite can be used to detect the fluorescent image of $\mathrm{A} \beta$ deposits in a mouse brain within 30 mins. This instant protocol is much cheaper and faster than conventional immunofluorescence staining technique clinically employed and provides an economic approach for detection of AD (Figure 2D). ${ }^{132}$

\section{The potential candidates for AD treatment Nanoparticle-conjugated quercetin}

Quercetin, a flavonoid existing in various foodstuffs, has antioxidative properties and increases GSH levels and antioxidant enzyme function. Extensive consideration has focused on increasing the intracellular GSH levels in many diseases, including AD. A $\beta$ 1-42 peptide when elevated in the brain of $\mathrm{AD}$ is associated with oxidative stress and neurotoxicity. ${ }^{133}$ In this study, nanoparticle-conjugated quercetin plays an important role in $\mathrm{AD}$ as the results demonstrated that neuronal cell death is attributable to metal-induced oxidative stress. Prominent among redox active metals initiating oxidative stress is $\mathrm{Cu}$ (II). Bioactive hybrid nanoparticles are developed for overcoming oxidative stress, and they are capable of working as host carriers for potent antioxidants such as the quercetin is detected in the release profiles of the 
loaded nanoparticle under oxidative stress in neuronal cultures. The bioactivity profile of quercetin nanoparticles in a neurodegenerative environment brought on by $\mathrm{Cu}$ (II) denotes the improved specificity of antioxidant reactivity counteracting oxidative stress and sets the stage for the development of molecular protection and preventive medical nanotechnology of relevance to neurodegenerative AD. ${ }^{134}$

\section{PLGA nanoparticles conjugated quercetin}

In vitro cytotoxicity studies of PLGA-conjugated quercetin (PLGA@QT)NPs inhibited and disassembled A $\beta_{42}$ fibrils neuroblastoma in SH-SY5Y cells; PLGA@QTNPs led to a concentration behavior with low cytotoxicity, illustrating that PLGA@ QTNPs can inhibit the neurotoxicity of $\mathrm{Zn}^{2+}-\mathrm{A} \beta_{42}$ system and improve the viability of neuron cells. Additionally, PLGA@ QTNPs are injected into APP/PS1 mice that led to partially abrogate memory impairments and also ameliorate cognition. Most interestingly, in vivo systemic toxicity of PLGA@QTNPs did not show any deterioration according to the histological analysis of significant organs in mice. Thereby, quercetin-based nanocarrier can augment therapeutic effects and subsequently reduce side effects, suggesting that the PLGA@QTNPs may be a potential candidate for $\mathrm{AD}$ treatment. ${ }^{135}$

\section{Conclusion}

Searching natural sources to be exploited as a complementary and alternative medicine such as nutraceuticals to meet urgent demand for the treatment of neurodegenerative disease has become a critical issue. Evidence base shows some natural compounds that contained the potential for AD treatment. However, the characters of natural compounds hindered the divergence including low water solubility, physicochemical instability, low aqueous stability, low bioavailability, low biocompatibility, rapid metabolism, and poor pharmacokinetics. This discrepancy leads to low efficacy of compounds in medical use. Nanoparticles possessing the unique properties become a new approach to improve this weakness. The nanocarrier formulations are featured in its particle size, adjustable component, and surface charges, which have been demonstrated to encapsulate commercial drugs or molecules. Hereby, nanoparticles conjugated with natural products could enhance the bioavailability and promote the efficacy of AD therapy when compared with naked drugs. Achievably, new delivery strategy creates and implements a new hope for drug discovery and is more effective for the application in the treatment of neurodegenerative diseases including AD. We warrant further studies in human subjects using nanocarriers conjugated with these potential natural compounds.

\section{Author contributions}

KRK, S-HY, and L-WW contributed to this review article writing. Both $\mathrm{C}-\mathrm{HL}$ and $\mathrm{C}-\mathrm{FW}$ executed as a supervisor to discuss and modify this manuscript. All authors contributed toward data analysis, drafting and revising the paper, gave final approval of the version to be published, and agree to be accountable for all aspects of the work.

\section{Disclosure}

The authors report no conflicts of interest in this work.

\section{References}

1. Brookmeyer R, Evans DA, Hebert L, et al. National estimates of the prevalence of Alzheimer's disease in the United States. Alzheimers Dement. 2011;7(1):61-73.

2. Iizuka T, Shoji M, Kawarabayashi T, et al. Intracellular generation of amyloid beta-protein from amyloid beta-protein precursor fragment by direct cleavage with beta- and gamma-secretase. Biochem Biophys Res Commun. 1996;218(1):238-242.

3. Nesi G, Sestito S, Digiacomo M, Rapposelli S. Oxidative stress, mitochondrial abnormalities and proteins deposition: multitarget approaches in Alzheimer's disease. Curr Top Med Chem. 2017;17(27): 3062-3079.

4. Pocernich CB, Lange ML, Sultana R, Butterfield DA. Nutritional approaches to modulate oxidative stress in Alzheimer's disease. Curr Alzheimer Res. 2011;8(5):452-469.

5. Norstrom E. Metabolic processing of the amyloid precursor protein new pieces of the Alzheimer's puzzle. Discov Med. 2017;23(127): 269-276.

6. Omtri RS, Thompson KJ, Tang X, et al. Differential effects of Alzheimer's disease A $\beta 40$ and 42 on Endocytosis and intraneuronal trafficking. Neuroscience. 2018;373:159-168.

7. Yoshiyama Y, Lee VM, Trojanowski JQ. Therapeutic strategies for tau mediated neurodegeneration. J Neurol Neurosurg Psychiatry. 2013; 84(7):784-795.

8. Alonso AD, Di Clerico J, Li B, et al. Phosphorylation of tau at Thr212, Thr231, and Ser262 combined causes neurodegeneration. J Biol Chem. 2010;285(40):30851-30860.

9. Nelson PT, Alafuzoff I, Bigio EH, et al. Correlation of Alzheimer disease neuropathologic changes with cognitive status: a review of the literature. J Neuropathol Exp Neurol. 2012;71(5):362-381.

10. Udeochu JC, Shea JM, Villeda SA. Microglia communication: parallels between aging and Alzheimer's disease. Clin Exp Neuroimmunol. 2016;7(2):114-125.

11. Ye SM, Johnson RW. Increased interleukin-6 expression by microglia from brain of aged mice. J Neuroimmunol. 1999;93(1-2): 139-148.

12. Ritzel RM, Patel AR, Pan S, et al. Age- and location-related changes in microglial function. Neurobiol Aging. 2015;36(6):2153-2163.

13. Lin HQ, Ho MT, Lau LS, Wong KK, Shaw PC, Wan DC. Antiacetylcholinesterase activities of traditional Chinese medicine for treating Alzheimer's disease. Chem Biol Interact. 2008;175(1-3): 352-354.

14. Haas C. Strategies, development, and pitfalls of therapeutic options for Alzheimer's disease. J Alzheimers Dis. 2012;28(2):241-281.

15. Becker RE, Greig NH. Alzheimer's disease drug development: old problems require new priorities. CNS Neurol Disord Drug Targets. 2008; 7(6):499-511.

16. Gosselet F, Saint-Pol J, Candela P, Fenart L. Amyloid- $\beta$ peptides, Alzheimer's disease and the blood-brain barrier. Curr Alzheimer Res. 2013;10(10):1015-1033.

17. Mawuenyega KG, Sigurdson W, Ovod V, et al. Decreased clearance of CNS beta-amyloid in Alzheimer's disease. Science. 2010; 330(6012):1774. 
18. van Assema DM, Lubberink M, Bauer M, et al. Blood-brain barrier P-glycoprotein function in Alzheimer's disease. Brain. 2012;135(Pt 1): 181-189.

19. Jedlitschky G, Vogelgesang S, Kroemer HK. MDR1-P-glycoprotein (ABCB1)-mediated disposition of amyloid- $\beta$ peptides: implications for the pathogenesis and therapy of Alzheimer's disease. Clin Pharmacol Ther. 2010;88(4):441-443.

20. Cascorbi I, Flüh C, Remmler C, et al. Association of ATP-binding cassette transporter variants with the risk of Alzheimer's disease. Pharmacogenomics. 2013;14(5):485-494.

21. Brenn A, Grube M, Peters M, et al. Beta-amyloid downregulates MDR1P-Glycoprotein (Abcb1) expression at the blood-brain barrier in mice. Int J Alzheimers Dis. 2011;2011:690121-690126.

22. Neuwelt EA, Bauer B, Fahlke C, et al. Engaging neuroscience to advance translational research in brain barrier biology. Nat Rev Neurosci. 2011;12(3):169-182.

23. Wolf A, Bauer B, Hartz AM. ABC Transporters and the Alzheimer's disease enigma. Front Psychiatry. 2012;3(54):54.

24. De Strooper B, Karran E. The cellular phase of Alzheimer's disease. Cell. 2016;164(4):603-615.

25. Prati F, Bottegoni G, Bolognesi ML, Cavalli A. BACE-1 inhibitors: from recent single-target molecules to multitarget compounds for Alzheimer's disease. J Med Chem. 2018;61(3):619-637.

26. Gold M. Phase II clinical trials of anti-amyloid $\beta$ antibodies: when is enough, enough? Alzheimers Dement. 2017;3(3):402-409.

27. Leng J, Qin HL, Zhu K, et al. Evaluation of multifunctional synthetic tetralone derivatives for treatment of Alzheimer's disease. Chem Biol Drug Des. 2016;88(6):889-898.

28. Xie S, Chen J, Li X, et al. Synthesis and evaluation of selegiline derivatives as monoamine oxidase inhibitor, antioxidant and metal chelator against Alzheimer's disease. Bioorg Med Chem. 2015;23(13):3722-3729.

29. Xia N, Liu L, Ning X, Lin L. Metallothioneins and synthetic metal chelators as potential therapeutic agents for removal of aberrant metal ions from metal-A $\beta$ species. Mini Rev Med Chem. 2014;14(3):271-281.

30. Bahramikia S, Yazdanparast R. Inhibition of human islet amyloid polypeptide or amylin aggregation by two manganese-salen derivatives. Eur J Pharmacol. 2013;707(1-3):17-25.

31. Dey A, Bhattacharya R, Mukherjee A, Pandey DK. Natural products against Alzheimer's disease: Pharmaco-therapeutics and biotechnological interventions. Biotechnol Adv. 2017;35(2):178-216.

32. Sandoval-Acuña C, Ferreira J, Speisky H. Polyphenols and mitochondria: an update on their increasingly emerging ROS-scavenging independent actions. Arch Biochem Biophys. 2014;559:75-90.

33. Beghyn T, Deprez-Poulain R, Willand N, Folleas B, Deprez B. Natural compounds: leads or ideas? Bioinspired molecules for drug discovery. Chem Biol Drug Des. 2008;72(1):3-15.

34. Garcia EJ, Oldoni TL, Alencar SM, Reis A, Loguercio AD, Grande RH. Antioxidant activity by DPPH assay of potential solutions to be applied on bleached teeth. Braz Dent J. 2012;23(1):22-27.

35. Farina N, Llewellyn D, Isaac M, Tabet N. Vitamin E for Alzheimer's dementia and mild cognitive impairment. Cochrane Database Syst Rev. 2017;4(4):CD002854

36. Sezgin Z, Dincer Y. Alzheimer's disease and epigenetic diet. Neurochem Int. 2014;78:105-116.

37. Zhang LN, Sun YJ, Pan S, et al. $\mathrm{Na}^{+}-\mathrm{K}^{+}$-ATPase, a potent neuroprotective modulator against Alzheimer disease. Fundam Clin Pharmacol. 2013;27(1):96-103.

38. Alam P, Chaturvedi SK, Siddiqi MK, et al. Vitamin $\mathrm{k} 3$ inhibits protein aggregation: implication in the treatment of amyloid diseases. Sci Rep. 2016;6:26759.

39. Mizwicki MT, Menegaz D, Zhang J, et al. Genomic and nongenomic signaling induced by $1 \alpha, 25(\mathrm{OH}) 2$-vitamin $\mathrm{D} 3$ promotes the recovery of amyloid- $\beta$ phagocytosis by Alzheimer's disease macrophages. J Alzheimers Dis. 2012;29(1):51-62.

40. Rigacci S, Stefani M. Nutraceuticals and amyloid neurodegenerative diseases: a focus on natural phenols. Expert Rev Neurother. 2015; 15(1):41-52.
41. Sawikr Y, Yarla NS, Peluso I, Kamal MA, Aliev G, Bishayee A. Chapter two - neuroinflammation in Alzheimer's disease: the preventive and therapeutic potential of polyphenolic nutraceuticals. In: Donev R, editor. Advances in Protein Chemistry and Structural Biology. Massachusetts: Academic Press; 2017:33-57.

42. Lodi A, Saha A, Lu X, et al. Combinatorial treatment with natural compounds in prostate cancer inhibits prostate tumor growth and leads to key modulations of cancer cell metabolism. NPJ Precis Oncol. 2017; 1(1):pii18.

43. Zasadil LM, Andersen KA, Yeum D, et al. Cytotoxicity of paclitaxel in breast cancer is due to chromosome missegregation on multipolar spindles. Sci Transl Med. 2014;6(229):229ra43.

44. Mclaurin J, Golomb R, Jurewicz A, Antel JP, Fraser PE. Inositol stereoisomers stabilize an oligomeric aggregate of Alzheimer amyloid beta peptide and inhibit abeta-induced toxicity. J Biol Chem. 2000; 275(24):18495-18502.

45. Mclaurin J, Kierstead ME, Brown ME, et al. Cyclohexanehexol inhibitors of Abeta aggregation prevent and reverse Alzheimer phenotype in a mouse model. Nat Med. 2006;12(7):801-808.

46. Lai AY, McLaurin J. Inhibition of amyloid-beta peptide aggregation rescues the autophagic deficits in the TgCRND8 mouse model of Alzheimer disease. Biochim Biophys Acta. 2012;1822(10):1629-1637.

47. Shanmugam MK, Rane G, Kanchi MM, et al. The multifaceted role of curcumin in cancer prevention and treatment. Molecules. 2015;20(2): 2728-2769.

48. Cianciulli A, Calvello R, Porro C, Trotta T, Salvatore R, Panaro MA. $\mathrm{PI} 3 \mathrm{k} /$ Akt signalling pathway plays a crucial role in the anti-inflammatory effects of curcumin in LPS-activated microglia. Int Immипорharmacol. 2016;36:282-290.

49. Esmaily H, Sahebkar A, Iranshahi M, et al. An investigation of the effects of curcumin on anxiety and depression in obese individuals: a randomized controlled trial. Chin J Integr Med. 2015;21(5):332-338.

50. Mattson MP, Son TG, Camandola S. Viewpoint: mechanisms of action and therapeutic potential of neurohormetic phytochemicals. Dose Response. 2007;5(3):174-186.

51. Ghosh S, Banerjee S, Sil PC. The beneficial role of curcumin on inflammation, diabetes and neurodegenerative disease: a recent update. Food Chem Toxicol. 2015;83:111-124.

52. Alam MN, Bristi NJ, Rafiquzzaman M. Review on in vivo and in vitro methods evaluation of antioxidant activity. Saudi Pharm J. 2013; 21(2):143-152.

53. Dohare P, Varma S, Ray M. Curcuma oil modulates the nitric oxide system response to cerebral ischemia/reperfusion injury. Nitric Oxide. 2008;19(1):1-11.

54. Dohare P, Garg P, Jain V, Nath C, Ray M. Dose dependence and therapeutic window for the neuroprotective effects of curcumin in thromboembolic model of rat. Behav Brain Res. 2008;193(2):289-297.

55. Zhang LJ, Wu CF, Meng XL, et al. Comparison of inhibitory potency of three different curcuminoid pigments on nitric oxide and tumor necrosis factor production of rat primary microglia induced by lipopolysaccharide. Neurosci Lett. 2008;447(1):48-53

56. Ataie A, Sabetkasaei M, Haghparast A, Moghaddam AH, Kazeminejad B. Neuroprotective effects of the polyphenolic antioxidant agent, curcumin, against homocysteine-induced cognitive impairment and oxidative stress in the rat. Pharmacol Biochem Behav. 2010;96(4):378-385.

57. Abe Y, Hashimoto S, Horie T. Curcumin inhibition of inflammatory cytokine production by human peripheral blood monocytes and alveolar macrophages. Pharmacol Res. 1999;39(1):41-47.

58. Bharti AC, Donato N, Aggarwal BB. Curcumin (diferuloylmethane) inhibits constitutive and IL-6-inducible STAT3 phosphorylation in human multiple myeloma cells. J Immunol. 2003;171(7):3863-3871.

59. Shi X, Zheng Z, Li J, et al. Curcumin inhibits A $\beta$-induced microglial inflammatory responses in vitro: involvement of ERK1/2 and p38 signaling pathways. Neurosci Lett. 2015;594:105-110.

60. Stankowska DL, Krishnamoorthy VR, Ellis DZ, Krishnamoorthy RR. Neuroprotective effects of curcumin on endothelin-1 mediated cell death in hippocampal neurons. Nutr Neurosci. 2017;20(5):273-283. 
61. Wang C, Zhang X, Teng Z, Zhang T, Li Y. Downregulation of PI3K Akt/mTOR signaling pathway in curcumin-induced autophagy in APP/ PS1 double transgenic mice. Eur J Pharmacol. 2014;740:312-320.

62. Zhang L, Fang Y, Xu Y, et al. Curcumin improves amyloid $\beta$-peptide (1-42) induced spatial memory deficits through BDNF-ERK signaling pathway. PLoS One. 2015;10(6):e0131525.

63. Xiong Z, Hongmei Z, Lu S, Yu L. Curcumin mediates presenilin-1 activity to reduce $\beta$-amyloid production in a model of Alzheimer's disease. Pharmacol Rep. 2011;63(5):1101-1108.

64. Tang M, Taghibiglou C. The mechanisms of action of curcumin in Alzheimer's disease. J Alzheimers Dis. 2017;58(4):1003-1016.

65. Ude C, Schubert-Zsilavecz M, Wurglics M. Ginkgo biloba extracts: a review of the pharmacokinetics of the active ingredients. Clin Pharmacokinet. 2013;52(9):727-749.

66. Augustin S, Rimbach G, Augustin K, Schliebs R, Wolffram S, Cermak R. Effect of a short- and long-term treatment with Ginkgo biloba extract on amyloid precursor protein levels in a transgenic mouse model relevant to Alzheimer's disease. Arch Biochem Biophys. 2009;481(2):177-182.

67. Abdel-Kader R, Hauptmann S, Keil U, et al. Stabilization of mitochondrial function by Ginkgo biloba extract (EGb 761). Pharmacol Res. 2007;56(6):493-502.

68. Bastianetto S, Zheng WH, Quirion R. The Ginkgo biloba extract (EGb 761) protects and rescues hippocampal cells against nitric oxideinduced toxicity. J Neurochem. 2000;74(6):2268-2277.

69. Shi C, Wu F, Xu J. H2O2 and PAF mediate Abeta1-42-induced Ca2+ dyshomeostasis that is blocked by EGb761. Neurochem Int. 2010; 56(8):893-905.

70. Yoshitake T, Yoshitake S, Kehr J. The Ginkgo biloba extract EGb 761(R) and its main constituent flavonoids and ginkgolides increase extracellular dopamine levels in the rat prefrontal cortex. $\mathrm{Br} J$ Pharmacol. 2010;159(3):659-668.

71. Jang JY, Min JH, Wang SB, et al. Resveratrol inhibits collagen-induced platelet stimulation through suppressing NADPH oxidase and oxidative inactivation of $\mathrm{SH} 2$ domain-containing protein tyrosine phosphatase-2. Free Radic Biol Med. 2015;89:842-851.

72. Cianciulli A, Dragone T, Calvello R, et al. IL-10 plays a pivotal role in anti-inflammatory effects of resveratrol in activated microglia cells Int Immunopharmacol. 2015;24(2):369-376.

73. Jhang KA, Park JS, Kim HS, Chong YH. Resveratrol ameliorates tau hyperphosphorylation at Ser396 site and oxidative damage in rat hippocampal slices exposed to vanadate: implication of ERK1/2 and GSK-3 $\beta$ signaling cascades. J Agric Food Chem. 2017;65(44):9626-9634.

74. Wang G, Chen L, Pan X, et al. The effect of resveratrol on beta amyloidinduced memory impairment involves inhibition of phosphodiesterase-4 related signaling. Oncotarget. 2016;7(14):17380-17392.

75. Feng X, Liang N, Zhu D, et al. Resveratrol inhibits $\beta$-amyloid-induced neuronal apoptosis through regulation of SIRT1-ROCK1 signaling pathway. PLoS One. 2013;8(3):e59888.

76. Ladiwala AR, Lin JC, Bale SS, et al. Resveratrol selectively remodels soluble oligomers and fibrils of amyloid Abeta into off-pathway conformers. J Biol Chem. 2010;285(31):24228-24237.

77. Wang J, Ho L, Zhao Z, et al. Moderate consumption of Cabernet Sauvignon attenuates Abeta neuropathology in a mouse model of Alzheimer's disease. FASEB J. 2006;20(13):2313-2320.

78. Ringman JM, Frautschy SA, Teng E, et al. Oral curcumin for Alzheimer's disease: tolerability and efficacy in a 24-week randomized, double blind, placebo-controlled study. Alzheimers Res Ther. 2012;4(5):43.

79. Quinn JF, Raman R, Thomas RG, et al. Docosahexaenoic acid supplementation and cognitive decline in Alzheimer disease: a randomized trial. JAMA. 2010;304(17):1903-1911.

80. Salloway S, Sperling R, Keren R, et al. A phase 2 randomized trial of ELND005, scyllo-inositol, in mild to moderate Alzheimer disease. Neurology. 2011;77(13):1253-1262.

81. Kaye J, Dekosky ST, Williamson JD. Ginkgo biloba for prevention of dementia: a randomized controlled trial. Arch Neurol. 2009;66(5): 652-654.
82. Dekosky ST, Williamson JD, Fitzpatrick AL, Snitz BE, O'Meara ES, Carlson MC. Ginkgo biloba for prevention of dementia: a randomized controlled trial. JAMA. 2008;300(19):2663-2670.

83. Yallapu MM, Nagesh PK, Jaggi M, Chauhan SC. Therapeutic applications of curcumin nanoformulations. Aaps J. 2015;17(6): 1341-1356.

84. Kankala RK, Tsai PY, Kuthati Y, Wei PR, Liu CL, Lee CH. Overcoming multidrug resistance through co-delivery of ROS-generating nano-machinery in cancer therapeutics. J Mater Chem B. 2017;5(7): 1507-1517.

85. Kankala RK, Liu CG, Chen AZ, et al. Overcoming multidrug resistance through the synergistic effects of hierarchical pH-sensitive, ROS-generating nanoreactors. ACS Biomater Sci Eng. 2017;3(10): 2431-2442.

86. Comoglu T, Arisoy S, Akkus ZB. Nanocarriers for effective brain drug delivery. Curr Top Med Chem. 2017;17(13):1490-1506.

87. Peer D, Karp JM, Hong S, Farokhzad OC, Margalit R, Langer R. Nanocarriers as an emerging platform for cancer therapy. Nat Nanotechnol. 2007;2(12):751-760.

88. Yeh YC, Creran B, Rotello VM. Gold nanoparticles: preparation, properties, and applications in bionanotechnology. Nanoscale. 2012;4(6): $1871-1880$

89. Murphy CJ, Gole AM, Stone JW, et al. Gold nanoparticles in biology: beyond toxicity to cellular imaging. Acc Chem Res. 2008;41(12): $1721-1730$

90. Huang X, El-Sayed MA. Gold nanoparticles: optical properties and implementations in cancer diagnosis and photothermal therapy. $J$ Adv Res. 2010;1(1):13-28.

91. Lohse SE, Murphy CJ. Applications of colloidal inorganic nanoparticles: from medicine to energy. J Am Chem Soc. 2012;134(38): $15607-15620$.

92. Jana NR, Earhart C, Ying JY. Synthesis of water-soluble and functionalized nanoparticles by silica coating. Chem Mater. 2007;19(21): 5074-5082.

93. Nagahama K, Utsumi T, Kumano T, Maekawa S, Oyama N, Kawakami J. Discovery of a new function of curcumin which enhances its anticancer therapeutic potency. Sci Rep. 2016;6:30962.

94. Manju S, Sreenivasan K. Gold nanoparticles generated and stabilized by water soluble curcumin-polymer conjugate: blood compatibility evaluation and targeted drug delivery onto cancer cells. $J$ Colloid Interface Sci. 2012;368(1):144-151.

95. Dey S, Sreenivasan K. Conjugating curcumin to water soluble polymer stabilized gold nanoparticles via $\mathrm{pH}$ responsive succinate linker. J Mater Chem B. 2015;3(5):824-833.

96. Palmal S, Maity AR, Singh BK, Basu S, Jana NR. Inhibition of amyloid fibril growth and dissolution of amyloid fibrils by curcumin-gold nanoparticles. Chemistry. 2014;20(20):6184-6191.

97. Jayaram DT, Shankar BH, Ramaiah D. Effective amyloid defibrillation by polyhydroxyl-substituted squaraine dyes. Chem Asian J. 2015 10(12):2689-2694.

98. Chandra B, Maity BK, Das A, Maiti S. Fluorescence quenching by lipid encased nanoparticles shows that amyloid- $\beta$ has a preferred orientation in the membrane. Chem Commun. 2018;54(56):7750-7753.

99. Peppas NA, Bures P, Leobandung W, Ichikawa H. Hydrogels in pharmaceutical formulations. Eur J Pharm Biopharm. 2000;50(1):27-46.

100. Soni G, Yadav KS. Nanogels as potential nanomedicine carrier for treatment of cancer: a mini review of the state of the art. Saudi Pharm J. 2016;24(2):133-139.

101. Yc Y, Funke W. Reactive microgels by emulsion polymerization of unsaturated polyester resins. Angew Makromol Chem. 1982;103(1): 187-202.

102. Liang FW, Funke W. Cross-linking self-emulsifying copolymerization of an unsaturated polyester and styrene. Macromolecules. 1996; 29(27):8650-8655.

103. Vinogradov SV, Bronich TK, Kabanov AV. Nanosized cationic hydrogels for drug delivery: preparation, properties and interactions with cells. Adv Drug Deliv Rev. 2002;54(1):135-147. 
104. Kabanov AV, Vinogradov SV. Nanogels as pharmaceutical carriers: finite networks of infinite capabilities. Angew Chem Int Ed Engl. 2009;48(30):5418-5429.

105. Wei X, Senanayake TH, Bohling A, Vinogradov SV. Targeted nanogel conjugate for improved stability and cellular permeability of curcumin: synthesis, pharmacokinetics, and tumor growth inhibition. Mol Pharm. 2014;11(9):3112-3122.

106. Jiang Z, Dong X, Liu H, Wang Y, Zhang L, Sun Y. Multifunctionality of self-assembled nanogels of curcumin-hyaluronic acid conjugates on inhibiting amyloid $\beta$-protein fibrillation and cytotoxicity. React Funct Polym. 2016;104:22-29.

107. Manju S, Sreenivasan K. Conjugation of curcumin onto hyaluronic acid enhances its aqueous solubility and stability. J Colloid Interface Sci. 2011;359(1):318-325.

108. Wang YJ, Pan MH, Cheng AL, et al. Stability of curcumin in buffer solutions and characterization of its degradation products. J Pharm Biomed Anal. 1997;15(12):1867-1876.

109. Kreuter J. Nanoparticles - a historical perspective. Int J Pharm. 2007; 331(1):1-10.

110. Couvreur P. Polyalkylcyanoacrylates as colloidal drug carriers. Crit Rev Ther Drug Carrier Syst. 1988;5(1):1-20.

111. Lu X-Y, Wu D-C, Li Z-J, Chen G-Q. Polymer nanoparticles. In: Villaverde A, editor. Progress in Molecular Biology and Translational Science. Massachusetts: Academic Press; 2011:299-323.

112. Shive MS, Anderson JM. Biodegradation and biocompatibility of PLA and PLGA microspheres. Adv Drug Deliv Rev. 1997;28(1):5-24.

113. Waghela BN, Sharma A, Dhumale S, Pandey SM, Pathak C. Curcumin conjugated with PLGA potentiates sustainability, anti-proliferative activity and apoptosis in human colon carcinoma cells. PLoS One. 2015;10(2):e0117526.

114. Yang X, Li Z, Wang N, et al. Curcumin-encapsulated polymeric micelles suppress the development of colon cancer in vitro and in vivo. Sci Rep. 2015;5:10322.

115. Gou M, Men K, Shi H, et al. Curcumin-loaded biodegradable polymeric micelles for colon cancer therapy in vitro and in vivo. Nanoscale. 2011;3(4):1558-1567.

116. Mulik RS, Mönkkönen J, Juvonen RO, Mahadik KR, Paradkar AR. ApoE3 mediated poly(butyl) cyanoacrylate nanoparticles containing curcumin: study of enhanced activity of curcumin against beta amyloid induced cytotoxicity using in vitro cell culture model. Mol Pharm. 2010;7(3):815-825.

117. Mathew A, Fukuda T, Nagaoka Y, et al. Curcumin loaded-PLGA nanoparticles conjugated with Tet-1 peptide for potential use in Alzheimer's disease. PLoS One. 2012;7(3):e32616.

118. Djiokeng Paka G, Doggui S, Zaghmi A, et al. Neuronal uptake and neuroprotective properties of curcumin-loaded nanoparticles on SK$\mathrm{N}-\mathrm{SH}$ cell line: role of poly(lactide-co-glycolide) polymeric matrix composition. Mol Pharm. 2016;13(2):391-403.

119. Doggui S, Sahni JK, Arseneault M, Dao L, Ramassamy C. Neuronal uptake and neuroprotective effect of curcumin-loaded PLGA nanoparticles on the human SK-N-SH cell line. J Alzheimers Dis. 2012; 30(2):377-392.

120. Paka GD, Ramassamy C. Optimization of curcumin-loaded PEG-PLGA nanoparticles by GSH functionalization: investigation of the internalization pathway in neuronal cells. Mol Pharm. 2017;14(1):93-106.

121. Bangham AD, Standish MM, Watkins JC. Diffusion of univalent ions across the lamellae of swollen phospholipids. J Mol Biol. 1965; 13(1):238-252.

122. Sessa G, Weissmann G. Incorporation of lysozyme into liposomes. A model for structure-linked latency. J Biol Chem. 1970;245(13): 3295-3301.
123. Gregoriadis $\mathrm{G}$. The carrier potential of liposomes in biology and medicine (second of two parts). N Engl J Med. 1976;295(14):765-770.

124. Jain S, Jain V, Mahajan SC. Lipid based vesicular drug delivery systems. Adv Pharm. 2014;2014(7):1-12.

125. Lazar AN, Mourtas S, Youssef I, et al. Curcumin-conjugated nanoliposomes with high affinity for $\mathrm{A} \beta$ deposits: possible applications to Alzheimer disease. Nanomedicine. 2013;9(5):712-721.

126. Mourtas S, Canovi M, Zona C, et al. Curcumin-decorated nanoliposomes with very high affinity for amyloid- $\beta 1-42$ peptide. Biomaterials. 2011;32(6):1635-1645.

127. Summerlin N, Soo E, Thakur S, Qu Z, Jambhrunkar S, Popat A. Resveratrol nanoformulations: challenges and opportunities. Int J Pharm. 2015;479(2):282-290.

128. Richard T, Pawlus AD, Iglésias ML, et al. Neuroprotective properties of resveratrol and derivatives. Ann N Y Acad Sci. 2011;1215(1): 103-108.

129. Frozza RL, Bernardi A, Hoppe JB, et al. Neuroprotective effects of resveratrol against $A \beta$ administration in rats are improved by lipid-core nanocapsules. Mol Neurobiol. 2013;47(3):1066-1080.

130. Loureiro J, Andrade S, Duarte A, et al. Resveratrol and grape extractloaded solid lipid nanoparticles for the treatment of Alzheimer's disease. Molecules. 2017;22(2):277.

131. Neves AR, Queiroz JF, Reis S. Brain-targeted delivery of resveratrol using solid lipid nanoparticles functionalized with apolipoprotein E. J Nanobiotechnology. 2016;14(1):27.

132. He XP, Deng Q, Cai L, et al. Fluorogenic resveratrol-confined graphene oxide for economic and rapid detection of Alzheimer's disease. ACS Appl Mater Interfaces. 2014;6(8):5379-5382.

133. Ansari MA, Abdul HM, Joshi G, Opii WO, Butterfield DA. Protective effect of quercetin in primary neurons against Abeta(1-42): relevance to Alzheimer's disease. J Nutr Biochem. 2009;20(4):269-275.

134. Nday CM, Halevas E, Jackson GE, Salifoglou A. Quercetin encapsulation in modified silica nanoparticles: potential use against $\mathrm{Cu}$ (II)induced oxidative stress in neurodegeneration. J Inorg Biochem. 2015;145:51-64.

135. Sun D, Li N, Zhang W, et al. Design of PLGA-functionalized quercetin nanoparticles for potential use in Alzheimer's disease. Colloids Surf B Biointerfaces. 2016;148:116-129.

136. Bianchetti A, Rozzini R, Trabucchi M. Effects of acetyl-L-carnitine in Alzheimer's disease patients unresponsive to acetylcholinesterase inhibitors. Curr Med Res Opin. 2003;19(4):350-353.

137. Hudson S, Tabet N. Acetyl-L-carnitine for dementia. Cochrane Database Syst Rev. 2003;2(2):CD003158.

138. Peng Y, Sun J, Hon S, et al. L-3-n-butylphthalide improves cognitive impairment and reduces amyloid-beta in a transgenic model of Alzheimer's disease. J Neurosci. 2010;30(24):8180-8189.

139. Pitt J, Thorner M, Brautigan D, Larner J, Klein WL. Protection against the synaptic targeting and toxicity of Alzheimer's-associated A $\beta$ oligomers by insulin mimetic chiro-inositols. FASEB J. 2013; 27(1):199-207.

140. Cascella M, Bimonte S, Muzio MR, Schiavone V, Cuomo A. The efficacy of Epigallocatechin-3-gallate (green tea) in the treatment of Alzheimer's disease: an overview of pre-clinical studies and translational perspectives in clinical practice. Infect Agent Cancer. 2017; $12: 36$.

141. Turner RS, Thomas RG, Craft S, et al. A randomized, doubleblind, placebo-controlled trial of resveratrol for Alzheimer disease. Neurology. 2015;85(16):1383-1391.

142. Yang G, Wang Y, Tian J, Liu JP. Huperzine A for Alzheimer's disease: a systematic review and meta-analysis of randomized clinical trials. PLoS One. 2013;8(9):e74916. 
International Journal of Nanomedicine

Dovepress

\section{Publish your work in this journal}

The International Journal of Nanomedicine is an international, peerreviewed journal focusing on the application of nanotechnology in diagnostics, therapeutics, and drug delivery systems throughout the biomedical field. This journal is indexed on PubMed Central, MedLine, CAS, SciSearch ${ }^{\circledR}$, Current Contents ${ }^{\circledR} /$ Clinical Medicine,
Journal Citation Reports/Science Edition, EMBase, Scopus and the Elsevier Bibliographic databases. The manuscript management system is completely online and includes a very quick and fair peer-review system, which is all easy to use. Visit http://www.dovepress.com/ testimonials.php to read real quotes from published authors.

Submit your manuscript here: http://www.dovepress.com/international-journal-of-nanomedicine-journal 